TRANSACTIONS OF THE

AMERICAN MATHEMATICAL SOCIETY

Volume 358, Number 4, Pages 1455-1468

S 0002-9947(05)04096-1

Article electronically published on November 18, 2005

\title{
LOW-DIMENSIONAL HOMOGENEOUS EINSTEIN MANIFOLDS
}

\author{
CHRISTOPH BÖHM AND MEGAN M. KERR
}

ABStract. We show that compact, simply connected homogeneous spaces up to dimension 11 admit homogeneous Einstein metrics.

A closed Riemannian manifold $\left(M^{n}, g\right)$ is called Einstein if the Ricci tensor of $g$ is a multiple of itself. This equation, called the Einstein equation, is a complicated system of second order partial differential equations, and at the present time no general existence results for Einstein metrics are known. However, there are results for many interesting classes of Einstein metrics, such as Kähler-Einstein metrics Yau, Tia, metrics with small holonomy group [Jo, Sasakian-Einstein metrics BoGa and homogeneous Einstein metrics [He], BWZ] (cf. [Bes, LeWa] for many more details and examples).

We investigate the Einstein equation for $G$-invariant metrics on compact homogeneous spaces. Due to this symmetry assumption the Einstein equation becomes a system of non-linear algebraic equations which in special instances can be solved explicitly, whereas in general this seems to be impossible. Yet there are general existence results on compact homogeneous Einstein manifolds [WZ2, BWZ, Bö], based on the variational characterization of Einstein metrics by the Hilbert action [Hi]. These results turn out to be very helpful in proving the following:

Theorem. Let $M^{n}$ be a compact, simply connected homogeneous space of dimension less than or equal to 11. Then $M^{n}$ admits a homogeneous Einstein metric.

This result is optimal, since the 12-dimensional compact, simply connected homogeneous space $S U(4) / S U(2)_{10}$ does not admit homogeneous Einstein metrics WZ2 (cf. Table 1).

A compact homogeneous Einstein manifold $\left(M^{n}, g\right)$ of dimension $n=2$ or 3 has constant sectional curvature. Simply connected compact homogeneous Einstein manifolds were classified by Jensen [Je1] in dimension $n=4$, and by Alekseevsky, Dotti and Ferraris $[\mathrm{ADF}$ in dimension $n=5$. It was shown by Nikonorov and Rodionov [NiRo] that all simply connected compact homogeneous manifolds in dimension $n=6$ admit a homogeneous Einstein metric, and in dimension $n=7$ by Castellani, Romans and Warner CRW]. In Ni2 Nikonorov determined all sevendimensional homogeneous Einstein metrics.

Compact homogeneous spaces $M^{n}$ can admit many homogeneous structures; that is, there may be many inequivalent presentations $M^{n}=G / H$ of $M^{n}$ as a homogeneous space. But for any presentation $M^{n}=\tilde{G} / \tilde{H}$ of a simply connected

Received by the editors December 17, 2003.

2000 Mathematics Subject Classification. Primary 53C30; Secondary 53C25.

The second author was partially supported by the Radcliffe Institute for Advanced Study and by the Clare Boothe Luce Foundation.

(C)2005 American Mathematical Society Reverts to public domain 28 years from publication 1455 
homogeneous space there is a canonical presentation $M^{n}=G / H$, where $G$ is a compact, connected, semisimple Lie subgroup of $\tilde{G}$. Since the universal cover of $G$ still acts transitively on $G / H$, we may assume that $G$ is simply connected. In what follows we restrict our attention to such presentations. Furthermore, we will list only irreducible Riemannian manifolds.

The symmetric metric on irreducible symmetric spaces is Einstein. The symmetric spaces which arise in dimensions up to 12 are $S^{n}=S O(n+1) / S O(n)$, $\mathbb{C} P^{n}=S U(n+1) / S(U(1) U(n))$, the Grassmannians $S O(p+q) / S O(p) S O(q)$, $S U(p+q) / S(U(p) U(q))$, and $S p(p+q) / S p(p) S p(q)$, then $S U(n) / S O(n)$, $S p(n) / U(n), S O(2 n) / U(n), S U(2 n) / S p(n)$, and finally $G_{2} / S O(4)$. More generally, every isotropy irreducible homogeneous space $G / H$ admits a unique $G$ invariant Einstein metric Wo. The isotropy irreducible examples which appear in dimensions up to 12 are $S^{6}=G_{2} / S U(3), S^{7}=\operatorname{Spin}(7) / G_{2}$, the Berger space $B^{7}=S O(5) / S O(3)_{10}$, and $G_{2} / S O(3)_{28}$. (To indicate the embedding, we use the Dynkin index of the corresponding subalgebra of type $\mathfrak{s u}(2)$ or $\mathfrak{u}(2)$.) Some spheres and projective spaces admit a smaller transitive Lie group and hence a larger space of homogeneous metrics; their Einstein metrics were classified by Ziller Zi. $S^{2 n+1}=S U(n+1) / S U(n)$ admits one $S U(n+1)$-invariant Einstein metric, $S^{4 n+3}=S p(n+1) / S p(n)$ admits two $S p(n+1)$-invariant Einstein metrics Je3, Zi], and $S p(n+1) / U(1) S p(n)=\mathbb{C} P^{2 n+1}$ admits two $S p(n+1)$-invariant Einstein metrics Zi]. Finally, the Stiefel manifold $V_{2}\left(\mathbb{R}^{n}\right)=S O(n) / S O(n-2)$ admits exactly one $S O(n)$-invariant Einstein metric [Ko], $\mathrm{BaHs}$.

In Table 1 we list the remaining simply connected, compact homogeneous spaces $M^{n}=G / H$ with $n \leq 12$, where $G$ is compact, connected, simply connected and simple. When we write " $=m$ " in the column Einstein metrics, we mean that there exist precisely $m G$-invariant Einstein metrics on $G / H$, up to isometry. When we write " $\geq m$ ", $G / H$ carries at least $m G$-invariant Einstein metrics, up to isometry. To the best of our knowledge, we provide all references as to who discovered those Einstein metrics first and who classified them.

The first class of examples in Table 1 is the homogeneous Kähler-Einstein manifolds $F_{3}=S U(3) / T^{2}, S p(2) / T^{2}, S U(4) / S(U(1) U(1) U(2)), G_{2}^{+}\left(\mathbb{R}^{7}\right)=G_{2} / U(2)_{1}$, $F_{4}=S U(4) / T^{3}$ and $G_{2} / T^{2}$. (Here $F_{3}$ and $F_{4}$ are the spaces of complex flags in $\mathbb{C}^{3}$ and $\mathbb{C}^{4}$, respectively.) As is well known, these spaces are coadjoint orbits. With the exception of $G_{2} / T_{2}$, all their homogeneous Einstein metrics are classified. Note that these metrics are not all Kähler.

The next class of examples in Table 1 is principal $S^{1}$-bundles over homogeneous Kähler-Einstein manifolds: The Aloff-Wallach spaces $W_{p, q}$ are principal $S^{1}$ bundles over $F_{3}$, and we obtain infinitely many homotopy types of homogeneous Einstein manifolds in dimension seven (cf. [AlWa, Wa1, [BGMR]). These spaces are classified up to homeomorphism and diffeomorphism [KS2, and it is known that there are pairs of Aloff-Wallach spaces which are homeomorphic but not diffeomorphic. The spaces $S p(2) / \Delta_{p, q} U(1)$ are principal $S^{1}$-bundles over $S p(2) / T^{2}$ and due to $\mathrm{Kl}]$ there are also infinitely many homotopy types among them. Finally, the spaces $S U(4) / S\left(\Delta_{p, q} U(1) U(2)\right)$ and $G_{2} / S U(2)_{1}$ are principal $S^{1}$-bundles over $S U(4) / S(U(1) U(1) U(2))$ and $G_{2} / U(2)_{1}$, respectively.

Another pair of examples is the compact Lie groups $S U(3) /\{\mathrm{id}\}$ and $S p(2) /\{\mathrm{id}\}$. The symmetric metric on the 8-dimensional compact Lie group $S U(3)$ is Einstein. Moreover, $S U(3)$ admits at least one other homogeneous Einstein metric, obtained 
TABle 1. $G$ simple

\begin{tabular}{|c|c|c|}
\hline$M^{n}$ & $G / H$ & Einstein metrics \\
\hline$\overline{F F_{3}}$ & 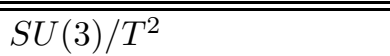 & $=2$ [Kosz, $[\mathrm{DN}]$ \\
\hline$W_{p, q}$ & $S U(3) / \Delta_{p, q} U(1)$ & $=2$ Wa1, $\mathrm{PP}, \mathrm{Ni2}$ \\
\hline \multirow[t]{4}{*}{$S U(3)$} & $S U(3) /\{\mathrm{id}\}$ & $\geq 2 \quad[\mathrm{Je} 2$ \\
\hline & $S p(2) / T^{2}$ & $=3$ Kosz, $\mathrm{Sa}$ \\
\hline & $S p(2) / \Delta_{p, q} U(1)$ & $\geq 1$ \\
\hline & $S U(4) / S(U(1) U(1) U(2))$ & $=3 \quad$ Kosz,, Ki \\
\hline$S p(2)$ & $S p(2) /\{\mathrm{id}\}$ & $\geq 2 \quad$ Je2 \\
\hline \multirow{8}{*}{$G_{2}^{+}\left(\mathbb{R}^{7}\right)$} & $G_{2} / U(2)_{1}$ & $=3$ Kosz, $\mathrm{Ke}$ \\
\hline & $G_{2} / U(2)_{3}$ & $=2 \quad$ DK2 \\
\hline & $S U(4) / S\left(\Delta_{p, q} U(1) U(2)\right)$ & $\geq 1$ \\
\hline & $S U(4) / U(2)_{2}$ & $=2 \quad$ Ni1 \\
\hline & $S U(4) / U(1) S O(3)_{4}$ & $=1 \quad$ WZ2 \\
\hline & $G_{2} / S U(2)_{1}$ & $\geq 2$ [Je3], Wa2] \\
\hline & $G_{2} / S U(2)_{3}$ & $\geq 2$ [Je3], Wa2] \\
\hline & $G_{2} / S O(3)_{4}$ & $=1$ DK1 \\
\hline$F_{4}$ & $S U(4) / T^{3}$ & $=3$ Kosz, $\mathrm{Sa}$ \\
\hline \multirow[t]{4}{*}{$V_{2}\left(\mathbb{C}^{4}\right)$} & $S U(4) / S U(2)_{1}$ & $\geq 1$ \\
\hline & $S U(4) / S U(2)_{2}$ & $\geq 1$ \\
\hline & $S U(4) / S O(3)_{4}$ & $\geq 1$ \\
\hline & $S U(4) / S U(2)_{10}$ & $=0 \quad$ WZ2 \\
\hline \multirow[t]{3}{*}{$S O(8) / U(4)$} & $S O(7) / U(3)$ & $=2 \quad \mathrm{WZ2}$ \\
\hline & $S p(3) / S p(1) S p(1) S p(1)$ & $=2$ WZ1, DK2 \\
\hline & $G_{2} / T^{2}$ & $\geq 1 \quad$ Kosz \\
\hline
\end{tabular}

via the canonical variation in the direction of the maximal subgroup $S O(3)$ Je2]. This second Einstein metric is not invariant under the adjoint action of $S U(3)$ on $\mathfrak{s u}(3)$, hence it comes with an infinite family of (isometric) Einstein metrics. This shows that in general the Einstein equation is not a generic system of $l$ polynomial equations with $l$ unknowns. The space of left-invariant metrics on $S U(3)$ is 36dimensional, thus finding all solutions to the Einstein equation is a highly nontrivial task.

For each of the spaces $S U(4) / U(1) S O(3)_{4}, S U(4) / S U(2)_{10}$ and $S O(8) / U(4)=$ $S O(7) / U(3)$, the space of $G$-invariant metrics of volume one is one-dimensional and the Einstein equations reduce to a quadratic equation in one variable. We note that for the above three spaces this equation has one, zero and two real solutions, respectively WZ2.

The remaining spaces are

$G_{2} / U(2)_{3}, S U(4) / U(2)_{2}, G_{2} / S U(2)_{3}, G_{2} / S O(3)_{4}$,

$S U(4) / S U(2)_{2}, V_{2}\left(\mathbb{C}^{4}\right)=S U(4) / S U(2)_{1}$,

$S U(4) / S O(3)_{4}$ and finally the space $S p(3) / S p(1) S p(1) S p(1)$ of quaternionic flags in $\mathbb{H} H^{3}$; 
TABLE 2. $G$ semisimple, $H_{s}$ embedded diagonally

\begin{tabular}{|l|l|l|}
\hline$M^{n}$ & $G / H$ & Einstein metrics \\
\hline \hline$F\left(\mathbb{C} P^{2} \times \mathbb{C} P^{2}, S^{3}\right)$ & $S U(3) \times S U(3) / \Delta S U(2)(U(1) \times U(1))$ & $=2$ \\
$F\left(S^{4} \times S^{4}, S^{3}\right)$ & $S p(2) \times S p(2) / \Delta S p(1)(S p(1) \times S p(1))$ & $=2$ \\
$F\left(\mathbb{C} P^{2} \times S^{4}, S^{3}\right)$ & $S U(3) \times S p(2) / \Delta_{1} S p(1)(U(1) \times S p(1))$ & $=2 \quad$ Wa2 \\
& $S U(3) \times S U(3) / \Delta U(2)$ & $\geq 1$ \\
& $S U(3) \times S U(3) / \Delta S U(2)\left(\Delta_{p, q} U(1)\right), p \neq q$ & $=0$ \\
& $S U(3) \times S U(3) / \Delta_{2} S U(2)(\{\mathrm{id}\} \times U(1))$ & $=2$ \\
& $S U(3) \times S p(2) / \Delta_{1} S p(1)(\{\mathrm{id}\} \times S p(1))$ & $=0$ \\
& $S U(3) \times S p(2) / \Delta_{2} S p(1)(\{\mathrm{id}\} \times S p(1))$ & $=2$ \\
\hline
\end{tabular}

the dimension of their spaces of $G$-invariant metrics of volume one ranges from three to thirteen. For some of them, we computed solutions to the Einstein equation explicitly; however, in the more complicated cases, we applied general existence results.

For the convenience of the reader, we indicate the embeddings of isotropy subgroups of $G_{2}$ and $S U(4)$ in Table 1, In $G_{2}, S U(2)_{1}<U(2)_{1}<S U(3) \cap S O(4)<$ $G_{2}, S U(2)_{3}<U(2)_{3}<S O(4)<G_{2}$ and $S O(3)_{4}<S O(4)<G_{2}$. In $S U(4)$, $S U(2)_{1}<U(2)_{1}<S U(3)<S U(4), S U(2)_{2}<U(2)_{2}<S p(2) \cap S O(4)<S U(4)$, $S O(3)_{4}<S O(4)<S U(4)$ and $S U(2)_{10}$ is maximal in $S p(2)<S U(4)$.

Now let us turn to the case when $G$ is simply connected, semisimple, but not simple. By the definition of our canonical presentation $M^{n}=G / H$, the projection of $H$ onto each simple factor is a proper subgroup. If $G / H=G_{1} / H_{1} \times G_{2} / H_{2}$, then existence of a $G$-invariant Einstein metric on $G / H$ is guaranteed if $G_{1} / H_{1}$ and $G_{2} / H_{2}$ admit $G_{i}$-invariant Einstein metrics. Therefore we can assume that $H$ lies "diagonally" in $G_{1} \times G_{2}$. There are two cases: For $H_{s}$ the semisimple part of $H$, either $H_{s}$ lies diagonally in $G_{1} \times G_{2}$ or it does not.

In Table 2 we list all examples for case 1 . First, we indicate the embeddings of some isotropy subgroups: In the third and seventh examples, $\operatorname{pr}_{1}\left(\Delta_{1} S p(1)\right)=$ $S U(2)$ and $\operatorname{pr}_{2}\left(\Delta_{1} S p(1)\right)=S p(1) \times\{$ id $\}$. In the sixth (resp. eighth) example, $\operatorname{pr}_{1}\left(\Delta_{2} S U(2)\right)=S O(3)$ and $\operatorname{pr}_{2}\left(\Delta_{2} S U(2)\right)=S U(2)\left(\right.$ resp. $\operatorname{pr}_{1}\left(\Delta_{2} S p(1)\right)=S O(3)$ and $\left.\operatorname{pr}_{2}\left(\Delta_{2} S p(1)\right)=S p(1) \times\{\operatorname{id}\}\right)$.

The first three homogeneous spaces in Table 2 are 11-dimensional homogeneous $S^{3}$-bundles over $\mathbb{C} P^{2} \times \mathbb{C} P^{2}, S^{4} \times S^{4}, \mathbb{C} P^{2} \times S^{4}$, respectively. The existence of two homogeneous Einstein metrics on these spaces is due to M. Wang Wa2. The family of 12-dimensional spaces $G_{p, q}:=S U(3) \times S U(3) / \Delta S U(2)\left(\Delta_{p, q} U(1)\right.$ ) (where $\Delta_{p, q} U(1)$ is embedded into $U(1) U(1)<S U(2) S U(2)$ with slope determined by $(p, q)$, for $p$ and $q$ relatively prime) provides the first examples of simply connected homogeneous torus bundles which do not admit $G$-invariant Einstein metrics. Note that $G_{1,1}=S U(3) \times S U(3) / \Delta U(2)$ admits a $G$-invariant Einstein metric by the Graph Theorem [BWZ. This is possible since for $G_{1,1}$ the dimension of the space of $G$-invariant metrics is larger than that for $G_{p, q}, p \neq q$. The following intriguing fact is due to W. Ziller: We have $H_{4}\left(G_{p, q}, \mathbb{Z}\right)=\mathbb{Z}_{6\left|p^{2}-q^{2}\right|}$ for $p^{2} \neq q^{2}$ and $H_{4}\left(G_{ \pm 1, \pm 1}, \mathbb{Z}\right)=\mathbb{Z}$. Hence in this case existence and non-existence of homogeneous Einstein metrics seems to be related to topological properties of the principal $S^{1}$-bundles under consideration. 
Finally, we describe all examples of case 2 above; that is, we assume that the center of $H$ lies diagonally in $G_{1} \times G_{2}$ but $H_{s}$ does not. Each of these spaces $M^{n}$ is a principal torus bundle over $B=G / H T$ with the presentation $M^{n}=G / H T^{\prime}$, $T^{\prime} \subsetneq T$. Since $\operatorname{dim} G / H \leq 12$, the base $G / H T$ turns out to be a product of homogeneous Kähler-Einstein manifolds with positive first Chern class.

The possible factors are $\mathbb{C} P^{1}=S U(2) / U(1), \mathbb{C} P^{2}=S U(3) / U(2), \mathbb{C} P^{3}=$ $S U(4) / U(3), \mathbb{C} P^{3}=S p(2) / U(1) S p(1), F_{3}=S U(3) / T^{2}, G_{2}^{+}\left(\mathbb{R}^{5}\right)=S p(2) / U(2)$, $\mathbb{C} P^{4}=S U(5) / U(4), G_{2}\left(\mathbb{C}^{4}\right)=S U(4) / S(U(2) U(2))$ and $S p(2) / T^{2}$, with the restriction that the sum of the dimension of the factors is bounded from the above by eleven. If the flag manifold $F_{3}$ or $S p(2) / T^{2}$ is not a factor, then existence of a $G$ invariant Einstein metric on $G / H$ follows from WZ3 and in dimension seven from the earlier work of physicists [CDF], [DFN]. For generic principal $S^{1}$-bundles over such bases, we also have uniqueness [WZ3. In the remaining cases, $B=F_{3} \times \mathbb{C} P^{1}$, $B=\mathbb{C} P^{1} \times S p(2) / T^{2}, B=F_{3} \times \mathbb{C} P^{2}$ and $B=F_{3} \times \mathbb{C} P^{1} \times \mathbb{C} P^{1}$, the existence of a $G$-invariant Einstein metric follows from [Bö].

From a topological and geometric point of view, many of these manifolds have been of great interest (cf. [KS1], WZ3]). For instance, the principal $S^{1}$-bundles over $\mathbb{C} P^{1} \times \mathbb{C} P^{1}$ are all diffeomorphic to $S^{2} \times S^{3}$, but as homogeneous spaces they are quite different. On $S^{2} \times S^{3}$ the moduli space of Einstein metrics has infinitely many components, all realized by homogeneous metrics.

This paper is organized as follows: In section 1 we recall some basic facts about compact homogeneous spaces. Then we give the classification of all simply connected compact homogeneous spaces $M^{n}$ up to dimension $n=12$, acted on by a compact, connected, simply connected and simple Lie group $G$. In the last section we provide a proof of our main theorem.

It is a pleasure to thank Wolfgang Ziller for helpful discussions.

\section{The Classification}

A connected, closed manifold $M^{n}$ is called a compact homogeneous space if there exists a compact group $G$ of diffeomorphisms acting transitively on $M^{n}$. Let $H$ denote the isotropy group at a point in $M^{n}$. Then the coset space $G / H$ is diffeomorphic to $M^{n}$. As is well known, both $G$ and $H$ are compact Lie groups. In what follows we will assume that $G / H$ is almost effective, i.e., $H$ contains no non-discrete normal subgroup of $G$. If the Lie algebra $\mathfrak{g}$ of $G$ is not semisimple, let $\mathfrak{g}=\mathfrak{z}(\mathfrak{g}) \oplus \mathfrak{g}_{s}$ denote the decomposition of $\mathfrak{g}$ into its center $\mathfrak{z}(\mathfrak{g})$ and its semisimple part $\mathfrak{g}_{s}$. Let $\left(G_{s}\right)_{0}$ denote the compact, connected subgroup of $G$ with Lie algebra $\mathfrak{g}_{s}$.

Lemma 1.1 ([0n, Prop. 9, p. 94]). Let $M^{n}=G / H$ be a compact, almost effective homogeneous space with finite fundamental group. Then $\left(G_{s}\right)_{0}$ acts transitively on $G / H$.

We obtain $G / H=\left(G_{s}\right)_{0} / H \cap\left(G_{s}\right)_{0}$ as manifolds.

Lemma 1.2. Let $G / H$ be a simply connected, compact homogeneous space. If $G$ is connected, then so is $H$.

We conclude that $H \cap\left(G_{s}\right)_{0}$ is connected if $M^{n}=G / H$ is simply connected. In order to define the canonical presentation of a compact homogeneous space $M^{n}=G / H$, one further reduction might be possible, by the following lemma. 
Lemma 1.3. Let $G / H$ be a simply connected compact homogeneous space, where $G$ is connected and semisimple, but not simple; that is, $\mathfrak{g}=\mathfrak{g}_{1} \oplus \cdots \oplus \mathfrak{g}_{p}$ for simple ideals $\mathfrak{g}_{i}, 1 \leq i \leq p$. Suppose that the projection of $\mathfrak{h}$ to $\mathfrak{g}_{1}$ is onto. Then the connected, semisimple Lie subgroup $G^{\prime}$ of $G$ with Lie algebra $\mathfrak{g}^{\prime}=\mathfrak{g}_{2} \oplus \cdots \oplus \mathfrak{g}_{p}$ still acts transitively on $G / H$.

Via this lemma, we obtain a connected and semisimple Lie group $G_{c a n}$ of $G$ acting transitively on a simply connected homogeneous space $M^{n}=G / H$ such that if $H_{c a n}=G_{c a n} \cap H$, the projection of the connected isotropy group $H_{\text {can }}$ to the simple factors of $G_{c a n}$ is not onto. We call the presentation $M^{n}=G_{c a n} / H_{c a n}$ the canonical presentation of the homogeneous space $G / H$.

In general the Lie group $G_{c a n}$ need not be simply connected. However, we may pass to the universal cover $\hat{G}_{c a n}$ of $G_{c a n}$ which still acts transitively on $M^{n}=G / H$.

Theorem 1.4. Let $M^{n}$ be a simply connected, compact homogeneous space. Then there exists a compact, connected, simply connected, semisimple Lie group $G=$ $G_{1} \times \cdots \times G_{p}, p \geq 1$, acting transitively and almost effectively on $M^{n}$ with connected isotropy group $H$, such that $\operatorname{pr}_{i}(H) \neq G_{i}$ for $1 \leq i \leq p$.

As is well known, the space of $G$-invariant metrics can be viewed as a subspace of the space $G_{c a n}$-invariant metrics. Moreover, the space of $\hat{G}_{c a n}$-invariant metrics agrees with the space of $G_{c a n}$-invariant metrics. This implies that by considering all $\hat{G}_{c a n}$-invariant metrics, for all canonical presentations $\hat{G}_{c a n} / \hat{H}_{c a n}$, we obtain all homogeneous metrics on the compact simply connected homogeneous space $M^{n}$. (Note that there still may be infinitely many canonical presentations of $M^{n}$.)

Remark 1.5. If $G$ is connected, compact and simply connected, then the second fundamental group of $G / H$ is finite if and only if $H$ is semisimple. Therefore, up to a fixed dimension, there are at most finitely many such simply connected, compact homogeneous spaces $M^{n}$. These have been classified by Klaus [Kl] up to dimension nine and by Kruggel $[\mathrm{Kr}$ up to dimension 12 (up to the last 2 spaces in Table 2).

Next, we investigate each compact, connected, simply connected semisimple Lie group $G$ and all its connected subgroups $H$, such that $\operatorname{dim} G / H \leq 12$.

Let $G$ be a connected, simply connected simple Lie group. The following number will be helpful: $\alpha(G):=\operatorname{dim} \mathfrak{g}-\operatorname{dim} \mathfrak{h}_{\max }$. We have

\begin{tabular}{ll|l|l}
$\mathfrak{g}$ & & $\alpha(G)$ & $\mathfrak{h}_{\max }$ \\
\hline $\mathfrak{s u}(r)$, & $r \geq 3, \neq 4$ & $2(r-1)$ & $\mathfrak{s u}(r-1) \oplus \mathbb{R}$ \\
$\mathfrak{s p}(r)$, & $r \geq 3$ & $4(r-1)$ & $\mathfrak{s p}(r-1) \oplus \mathfrak{s p}(1)$ \\
$\mathfrak{s o}(r)$, & $r \geq 3, r \neq 4$ & $r-1$ & $\mathfrak{s o}(r-1)$ \\
$\mathfrak{g}_{2}$ & & 6 & $\mathfrak{s u}(3)$ \\
$\mathfrak{f}_{4}$ & & 16 & $\mathfrak{s} \mathfrak{l}(9)$ \\
$\mathfrak{e}_{6}$ & & 26 & $\mathfrak{f}_{4}$ \\
$\mathfrak{e}_{7}$ & & 54 & $\mathfrak{e}_{6} \oplus \mathbb{R}$ \\
$\mathfrak{e}_{8}$ & & 112 & $\mathfrak{e}_{7} \oplus \mathfrak{s u}(2)$
\end{tabular}

Note that $\mathfrak{s o}(3)=\mathfrak{s u}(2), \mathfrak{s o}(4)=\mathfrak{s u}(2) \oplus \mathfrak{s u}(2), \mathfrak{s o}(5)=\mathfrak{s p}(2)$, and $\mathfrak{s o}(6)=\mathfrak{s u}(4)$ (cf. $[\mathrm{Ad}]$ ).

We start by finding all simple $G$ for which $\alpha(G) \leq 12$. For each such group, we list all the subgroups $H<G$ for which $\operatorname{dim}(G / H) \leq 12$ (see Dy1]). 
Case 1: $G=S U(N), N=2, \ldots, 7$.

$N=2$ : We get two subgroups of $S U(2): U(1)$ and $\{\operatorname{id}\}$.

$N=3$ : We have two maximal subgroups of $S U(3): U(2)=S(U(1) U(2))$ and $S O(3)$, and within $U(2), S U(2), T^{2}=U(1) U(1), \Delta_{p, q} U(1)$, and $\{\mathrm{id}\}$.

$N=4$ : We have four maximal subgroups of $S U(4): U(3)=S(U(1) U(3))$, $S(U(2) U(2)), S p(2)$, and $S O(4)$. Their subgroups $H$ with $\operatorname{dim}(H) \geq 3$ are given by

(a) $S U(3)<U(3)$,

(b) $S U(2)_{1} S U(2)_{1}<S\left(U(2)_{1} U(2)_{1}\right)$ (equiv. to $S p(1)_{1} S p(1)_{1}<S p(2)$ ),

(c) $S\left(U(1) U(1) U(2)_{1}\right)=S(U(1) U(3)) \cap S\left(U(2)_{1} U(2)_{1}\right)$,

(d) $S\left(\Delta_{p, q} U(1) U(2)_{1}\right)<S\left(U(1) U(1) U(2)_{1}\right)$,

(e) $U(2)_{2}<S p(2)$ (equiv. to $U(2)_{2}<S O(4)$ ),

(f) $U(1) S O(3)_{4}$,

(g) $S U(2)_{1}<U(2)_{1}<S U(3)$ (equiv. to $S U(2)_{1}<S U(2)_{1} S U(2)_{1}$ and $S p(1)_{1}$ $\left.<S p(1)_{1} S p(1)_{1}\right)$

(h) $S U(2)_{2}<U(2)_{2}$ (equiv. to $\Delta S p(1)_{1}<S p(1)_{1} S p(1)_{1}, \Delta S U(2)_{1}<$ $\left.S U(2)_{1} S U(2)_{1}\right)$

(i) $S O(3)_{4}<S O(4)$,

(j) $S U(2)_{10}<S p(2)$ (maximal, see Dy2, Table 13),

(k) $T^{3}$.

$N=5$ : We have three maximal subgroups of $S U(5): U(4)=S(U(1) U(3))$, $S(U(2) U(3)), S O(5)$. We need $\operatorname{dim}(H) \geq 12$, which eliminates $S O(5)$. The only non-maximal subgroup with sufficiently large dimension is $S U(4)<U(4)$.

$N=6: S U(6)$ has six maximal subgroups. We want only those subgroups of $S U(6)$ with $\operatorname{dim}(H) \geq 23$; this leaves one maximal subgroup $U(5)=S(U(1) U(5))$, and also $S U(5)<U(5)$.

$N=7$ : The only subgroup of $S U(7)$ with $\operatorname{dim}(H) \geq 36$ is $U(6)$.

Case 2: $G=S p(N)$ for $N=2,3,4$.

$N=2$ : The maximal subgroups of $S p(2)$ are $U(2), S p(1) S p(1)$, and $S U(2)$. We also have their subgroups:

(a) $U(1) S p(1)<S p(1) S p(1)$,

(b) $S p(1)<S p(1) S p(1)$,

(c) $S U(2)<U(2)$ (equiv. to $\Delta S p(1)<S p(1) S p(1)$ ),

(d) $T^{2}=U(1) U(1)$,

(e) $\Delta_{k, l} U(1)<U(1) U(1)$,

(f) $\{$ id $\}$.

$N=3: S p(3)$ has four maximal subgroups: $U(3), S p(1) S p(2), S p(1) S O(3)$, and $S U(2)$. We want only the subgroups with $\operatorname{dim}(H) \geq 9$. This leaves us with $U(3)$, $S p(1) S p(2), U(1) S p(2), S p(2)$, and $S p(1) S p(1) S p(1)$.

$N=4$ : The only subgroup of $S p(4)$ satisfying $\operatorname{dim}(H) \geq 24$ is $S p(1) S p(3)$.

Case 3: $G=S O(N)$ for $N=7, \ldots, 13$.

$N=7$ : The group $S O(7)$ has four maximal subgroups: $S O(6), S O(2) S O(5)$, $S O(3) S O(4)$ and $G_{2}$. Since we are only interested in those subgroups of $S O(7)$ with $\operatorname{dim}(H) \geq 9$, we are left with $U(3)$ and $S O(5)=S O(2) S O(5) \cap S O(6)$. 
$N=8$ : The group $S O(8)$ has five maximal subgroups. Since we need $\operatorname{dim}(H) \geq 16$, this leaves $S O(2) S O(6)$ (equivalent to $U(4)$ ) and $S O(7)$ of dimension 21. Then $S O(7)$ has no proper subgroups of dimension at least 17 .

$N=9,10,11,12,13$ : The only quotients with dimension less than or equal to 12 are the spheres: $S O(n) / S O(n-1)$.

Case 4: $G=G_{2}$. This is the only exceptional Lie group with $\alpha(G) \leq 12$. The group $G_{2}$ has three maximal subgroups: $S U(3), S O(4)$ and $S O(3)_{28}$ (see Dy2, Table 14). Their subgroups with $\operatorname{dim} H \geq 2$ are given by:

(a) $U(2)_{1}<S U(3) \cap S O(4)$,

(b) $U(2)_{3}<S O(4)($ not in $S U(3))$,

(c) $S U(2)_{1}<U(2)_{1}$,

(d) $S U(2)_{3}<U(2)_{3}$,

(e) $S O(3)_{4}<S O(4)$,

(f) $T^{2}$.

This completes the classification for the case $G$ simple.

Now let us turn to the semisimple groups. Let $M^{n}=G / H$ be a connected, compact homogeneous space written in canonical form: $G=G_{1} \times \cdots \times G_{p}$, where each $G_{i}$ is compact, simple, simply connected, and $p \geq 2$. We have $G_{i} \cap H<$ $\operatorname{pr}_{i}(H)<G_{i}$. If $n \leq 10$, then for some $k \geq 1, G / H$ can be written $G / H=$ $\left(G_{1} \times \cdots \times G_{p}\right) / \Delta T^{k}\left(H_{1} \times \cdots \times H_{p}\right)$. To see this, we follow the method used in [Kl]. Consider the fibration

$$
\left(\operatorname{pr}_{1}(H) \times \cdots \times \operatorname{pr}_{p}(H)\right) / H \rightarrow G / H \rightarrow G_{1} / \operatorname{pr}_{1}(H) \times \cdots \times G_{p} / \operatorname{pr}_{p}(H) .
$$

Let $f=\operatorname{dim}\left(\left(\operatorname{pr}_{1}(H) \times \cdots \times \operatorname{pr}_{p}(H)\right) / H\right), b_{i}=\operatorname{dim}\left(G_{i} / \operatorname{pr}_{i}(H)\right)$. Then $\operatorname{dim} G / H=$ $f+\left(b_{1}+\cdots+b_{p}\right)$. We know $f \geq 1$, and $b_{i} \geq 2, b_{i} \neq 3$. In order for one of the simple factors of the semisimple part of $H$ to lie diagonally in $G$, we must have $b_{1}, b_{2} \geq 4$, and $f \geq 3$. This implies $\operatorname{dim} G / H \geq 11$.

In dimension $11=3+4+4$ we obtain the following possibilities for $G / H$ :

(a) $S U(3) \times S U(3) / \Delta S U(2)(U(1) \times U(1))$,

(b) $S p(2) \times S p(2) / \Delta S p(1)(S p(1) \times S p(1))$,

(c) $S U(3) \times S p(2) / \Delta_{1} S p(1)(U(1) \times S p(1))$.

In dimension $12=3+4+5$ we get

(a) $S U(3) \times S U(3) / \Delta S U(2)\left(\Delta_{p, q} U(1)\right)$,

(b) $S U(3) \times S U(3) / \Delta_{1} S U(2)(\{$ id $\} \times U(1))$,

(c) $S U(3) \times S p(2) / \Delta_{1} S p(1)(\{$ id $\} \times S p(1))$,

(d) $S U(3) \times S p(2) / \Delta_{2} S p(1)(\{\mathrm{id}\} \times S p(1))$.

The diagonal embeddings of $S U(2)$ and $S p(1)$, respectively, are described in the Introduction.

\section{Existence OF Einstein METRICS}

Let $G / H$ be a compact homogeneous space and let $Q$ denote an $A d(G)$-invariant scalar product on $\mathfrak{g}$. Choose $\mathfrak{m}$ the $Q$-orthogonal complement to $\mathfrak{h}$ in $\mathfrak{g}$. As is well known, every $G$-invariant metric on $G / H$ is uniquely determined by an $A d(H)$ invariant scalar product on $\mathfrak{m}$. For any $G$-invariant metric $\langle\cdot, \cdot\rangle$ on $G / H$, there exists a corresponding decomposition $\mathfrak{m}=\mathfrak{m}_{1} \oplus \cdots \oplus \mathfrak{m}_{p}$ of $\mathfrak{m}$ into $A d(H)$-irreducible summands, such that $\langle\cdot, \cdot\rangle$ is diagonal with respect to $Q$. That is, we have

$$
\langle\cdot, \cdot\rangle=x_{1} Q\left|\mathfrak{m}_{1} \perp \cdots \perp x_{p} Q\right| \mathfrak{m}_{p}
$$


with $x_{1}, \ldots, x_{p}>0$. The scalar curvature for $\langle\cdot, \cdot\rangle$ is given in WZ2]:

$$
S=\frac{1}{2} \sum_{i} \frac{d_{i} b_{i}}{x_{i}}-\frac{1}{4} \sum_{i, j, k}[i j k] \frac{x_{i}}{x_{j} x_{k}} .
$$

In this formula, for each $i,-B\left|\mathfrak{m}_{i}=b_{i} Q\right| \mathfrak{m}_{i}$, where $B$ denotes the Killing form, and $d_{i}=\operatorname{dim} \mathfrak{m}_{i}$; the triple $[i j k]=\sum Q\left(\left[X_{\alpha}, X_{\beta}\right], X_{\gamma}\right)^{2}$ is summed over $\left\{X_{\alpha}\right\}$, $\left\{X_{\beta}\right\}$, and $\left\{X_{\gamma}\right\}, Q$-orthonormal bases for $\mathfrak{m}_{i}, \mathfrak{m}_{j}$, and $\mathfrak{m}_{k}$, respectively. Recall that $G$-invariant Einstein metrics on $G / H$ are characterized variationally as the critical points of the scalar curvature function $S$ restricted to the set of $G$-invariant metrics of volume 1 .

If the above-mentioned decomposition of $\mathfrak{m}$ is unique up to order, e.g. if $\operatorname{rk} G=$ rk $H$, then the Euler-Lagrange equation can be written down easily. The metric $\langle\cdot, \cdot\rangle$ is Einstein with Einstein constant $\lambda \geq 0$ (cf. [Bo]), if and only if for all $1 \leq i \leq p$,

$$
\frac{b_{i}}{2 x_{i}}-\frac{1}{4 d_{i}} \sum_{j, k}[i j k] \frac{2 x_{k}^{2}-x_{i}^{2}}{x_{i} x_{j} x_{k}}=\lambda .
$$

For certain homogeneous spaces this equation can be solved explicitly, and we will make use of this approach in proving our main theorem. We will also apply the following two general existence results on homogeneous Einstein metrics.

First, we describe a consequence of the Graph Theorem [BWZ].

Proposition 2.1 ([BWZ). Let $G / H$ be a compact homogeneous space with both $G$ and $H$ connected. Suppose that there exists a connected, semisimple intermediate subgroup $K$, such that $H$ is maximal in $K$ and $K$ is maximal in $G$. If there exists a further connected subgroup $L$ with $H<L<G$ not isomorphic to $K$, then $G / H$ admits a $G$-invariant Einstein metric.

Next, let us turn to the second general existence result. Suppose that $G / H$ is simply connected and compact, such that $G$ is compact, connected, simply connected and semisimple. Recall that $H$ is connected. As described in [Bö], we can assign a simplicial complex $\hat{\Delta}_{G / H}$ (the extended simplicial complex of $\left.G / H\right)$ to such a homogeneous space as follows: Let $T$ be a maximal torus of a compact complement of $H$ in the identity component of the normalizer $N_{G}(H)$ of $H$ in $G$. Then $T H$ is a connected, compact, proper subgroup of $G$ and there exist at most finitely many subalgebras of $\mathfrak{g}$ lying properly between $\mathfrak{t} \oplus \mathfrak{h}$ and $\mathfrak{g}$. These subalgebras are partially ordered by the inclusion relation. The extended simplicial complex $\hat{\Delta}_{G / H}$ of $G / H$ is the corresponding flag complex: Vertices correspond to flags of length one, edges to flags of length two, and so on. The subcomplex $\Delta_{G / H}$ of $\hat{\Delta}_{G / H}$ given by all flags of subalgebras generated by minimal subalgebras is called the simplicial complex of $G / H$. Note that both of these simplicial complexes are independent of the choice of $T$. In general, $\hat{\Delta}_{G / H}$ and $\Delta_{G / H}$ are homotopy equivalent but not homeomorphic.

Theorem $2.2(\overline{\mathrm{B}}])$. Let $G / H$ be a compact homogeneous space with both $G$ and $H$ connected. If the simplicial complex $\Delta_{G / H}$ of $G / H$ is not contractible, then $G / H$ admits a $G$-invariant Einstein metric. 
The empty set is by definition non-contractible. The following obvious property of the simplicial complex turns out to be extremely helpful.

Proposition $2.3([\mathrm{Bö}])$. Let $G / H$ be a simply connected, compact homogeneous space with both $G$ and $H$ connected. Let $T$ be a maximal torus of a compact complement of $H$ in the identity component of the normalizer $N_{G}(H)$ of $H$ in $G$. Then $\Delta_{G / H}=\Delta_{G / T H}$.

Furthermore, the simplicial complex obeys the following "product formula". Here $X * Y$ denotes the join of $X$ and $Y$. Note that $X * \emptyset=X$ and $\emptyset * \emptyset=\emptyset$.

Theorem $2.4([\mathrm{~B} \ddot{0}])$. Let $G / H$ be a simply connected, compact homogeneous space with both $G$ and $H$ connected. If $G=G_{1} \times \cdots \times G_{p}$ and $H=H_{1} \times \cdots \times H_{p}, H_{i}<G_{i}$, then the simplicial complex $\Delta_{G / H}$ is homeomorphic to $\Delta_{G_{1} / H_{1}} * \cdots * \Delta_{G_{p} / H_{p}} * S^{p-2}$.

We are now ready to prove our main theorem. We start with the case when $G$ is simple, by passing through Table 1 . There are five spaces which are not covered by the references provided there. By Proposition 2.3 we have $\Delta_{S p(2) / \Delta_{p, q} U(1)}=$ $\Delta_{S p(2) / T^{2}}$. Since the simplicial complex $\Delta_{S p(2) / T^{2}}$ is not connected, by Theorem 2.2 the existence of at least one $S p(2)$-invariant Einstein metric follows. Similarly, we have $\Delta_{S U(4) / S\left(\Delta_{p, q} U(1) U(2)\right)}=\Delta_{S U(4) / S(U(1) U(1) U(2))}$ and again existence of at least one $S U(4)$-invariant Einstein metric follows. By the same reasoning the complex Stiefel manifold $S U(4) / S U(2)_{1}$ admits an $S U(4)$-invariant Einstein metric. By Theorem 2.2. $S U(4) / S U(2)_{2}$ admits an $S U(4)$-invariant Einstein metric, since $\Delta_{S U(4) / S U(2)_{2}}=\Delta_{S U(4) / U(2)_{2}}$ is disconnected. Finally, with $K=S O(4)$ and $L=S U(3)$, it follows from Proposition 2.1 that $S U(4) / S O(3)_{4}$ carries an $S U(4)-$ invariant Einstein metric.

Using equation (目) and Maple, one sees that $S U(4) / U(2)_{2}$ carries exactly two Einstein metrics; we extend the result of [Ni1] for this space. This completes the proof of the main theorem in case $G$ is simple.

Now let us turn to the case $G$ semisimple, not simple. First, we consider the case when $G / H$ is one of our eight spaces with a nonabelian diagonal component in $H$. The first three examples were all proved to admit exactly two homogeneous Einstein metrics in Wa2]. We will start with the fifth space: $G / H=S U(3) \times$ $S U(3) / \Delta S U(2)\left(\Delta_{p, q} U(1)\right)$. The fourth space is a special case of this, with $p=q$.

We take $Q(X, Y)=-\frac{1}{2} \operatorname{tr}(X Y)$, a multiple of the Killing form. Let $G=G_{1} \times G_{2}$. We have an intermediate subgroup $K=K_{1} \times K_{2}=U(2) \times U(2)$, such that $H<$ $K<G$, and each $K_{i}<G_{i}$. We see that $\mathfrak{m}$ decomposes into four irreducible summands, pairwise inequivalent as long as $p \neq q$ : Let $\mathfrak{m}_{1}$ be the tangent space to $G_{1} / K_{1}$ and let $\mathfrak{m}_{2}$ be the tangent space to $G_{2} / K_{2}$. That is, $\mathfrak{s u}(3)_{1}=\mathfrak{u}(2)_{1} \oplus \mathfrak{m}_{1}$, and $\mathfrak{s u}(3)_{2}=\mathfrak{u}(2)_{2} \oplus \mathfrak{m}_{2}$. Next, $\mathfrak{m}_{3}=\mathfrak{s u}(2)_{1} \oplus \mathfrak{s u}(2)_{2} \ominus \Delta \mathfrak{s u}(2)$, the $Q$-orthogonal complement to $\Delta \mathfrak{s u}(2)$ in $\mathfrak{s u}(2)_{1} \oplus \mathfrak{s u}(2)_{2}$. Similarly, $\mathfrak{m}_{4}=\mathfrak{u}(1)_{1} \oplus \mathfrak{u}(1)_{2} \ominus \Delta_{p, q} \mathfrak{u}(1)$, the complement to $\Delta_{p, q} \mathfrak{u}(1)$ in $\mathfrak{u}(1)_{1} \oplus \mathfrak{u}(1)_{2}$.

Since $G_{1} / K_{1}, G_{2} / K_{2}$ and $K / H$ are symmetric spaces, we know $\left[\mathfrak{m}_{1}, \mathfrak{m}_{1}\right] \subset \mathfrak{k}_{1}$, $\left[\mathfrak{m}_{2}, \mathfrak{m}_{2}\right] \subset \mathfrak{k}_{2},\left[\mathfrak{m}_{3}, \mathfrak{m}_{3}\right] \subset \Delta \mathfrak{s u}(2)$ and $\left[\mathfrak{m}_{4}, \mathfrak{m}_{4}\right]=\left[\mathfrak{m}_{3}, \mathfrak{m}_{4}\right]=0$. Note that $\left[\mathfrak{m}_{1}, \mathfrak{m}_{2}\right]=$ 0 . We conclude that the only non-zero symmetric triples are [114], [224], [113] and [223].

We find $[113]=[223]=6$. The symmetric triples involving $\mathfrak{m}_{4}$ depend on the embedding of $\Delta_{p, q} U(1)$ in $U(1) U(1):[114]=12 \lambda^{2}$ and [224] $=12\left(1-\lambda^{2}\right)$, for some $\lambda=\lambda(p, q)$ with $\lambda^{2} \leq 1$. We have $d_{1}=d_{2}=4, d_{3}=3, d_{4}=1$, while $b_{i}=12$ for all 
$i$. Hence, the Einstein equation is given by the system

$$
\begin{aligned}
\frac{3 \lambda^{2} x_{4}}{x_{1}^{2}}+\frac{3\left(1-\lambda^{2}\right) x_{4}}{x_{2}^{2}} & =\frac{4}{x_{3}}+\frac{x_{3}}{2 x_{1}^{2}}+\frac{x_{3}}{2 x_{2}^{2}}=\frac{6}{x_{1}}-\frac{3 \lambda^{2} x_{4}}{2 x_{1}^{2}}-\frac{3 x_{3}}{4 x_{1}^{2}} \\
& =\frac{6}{x_{2}}-\frac{3\left(1-\lambda^{2}\right) x_{4}}{2 x_{2}^{2}}-\frac{3 x_{3}}{4 x_{2}^{2}} .
\end{aligned}
$$

Denote these four terms by $I, I I, I I I$ and $I V$, respectively. We get $2 \cdot I I I+2 \cdot I V+$ $I=5 \cdot I I$, an equation independent of $\lambda$. If we let $\alpha=\frac{x_{3}}{x_{1}}, \beta=\frac{x_{3}}{x_{2}}$ and simplify, then we obtain $4\left(\alpha^{2}+\beta^{2}\right)+20-12(\alpha+\beta)=0$, which has no real solutions. This shows that $S U(3) \times S U(3) / \Delta S U(2)\left(\Delta_{p, q} U(1)\right)$ carries no $S U(3) \times S U(3)$-invariant Einstein metrics, as long as $p \neq q$.

However, in the case $p=q, \mathfrak{m}_{1}$ and $\mathfrak{m}_{2}$ are equivalent summands in the isotropy representation, and so there is in fact a larger family of homogeneous metrics. We can write our homogeneous space as $S U(3) \times S U(3) / \Delta U(2)$. Then $H=\Delta U(2)$ is maximal in $K=\Delta S U(3)$, which is itself maximal in $G=S U(3) \times S U(3)$. Since $H<L=U(2) \times U(2)$, the existence of a $G$-invariant Einstein metric follows from Proposition 2.1. Note that from the computation above we know that no metric which is diagonal with respect to the decomposition above is Einstein.

The next space we consider is $S U(3) \times S U(3) / \Delta_{2} S U(2)(\{\mathrm{id}\} \times U(1))$, where $\operatorname{pr}_{1}\left(\Delta_{2} S U(2)\right)=S O(3)$ and $\operatorname{pr}_{2}\left(\Delta_{2} S U(2)\right)=S U(2)$. There are three irreducible summands in the isotropy representation. We have an intermediate subgroup $K=$ $K_{1} \times K_{2}=S O(3) \times U(2)$. Using this, we let $\mathfrak{m}_{1}$ be the $Q$-orthogonal complement to $\mathfrak{k}_{1}$ in $\mathfrak{s u}(3)_{1}$, while $\mathfrak{m}_{2}$ is the complement to $\mathfrak{k}_{2}$ in $\mathfrak{s u}(3)_{2}$. This leaves us with $\mathfrak{m}_{3}=\mathfrak{s u}(2) \oplus \mathfrak{s u}(2) \ominus \Delta \mathfrak{s u}(2)$. As in the example above, since $G_{1} / K_{1}$ and $G_{2} / K_{2}$ are symmetric spaces, we know $\left[\mathfrak{m}_{1}, \mathfrak{m}_{1}\right] \subset \mathfrak{k}_{1},\left[\mathfrak{m}_{2}, \mathfrak{m}_{2}\right] \subset \mathfrak{k}_{2}$. We also know $\left[\mathfrak{m}_{3}, \mathfrak{m}_{3}\right] \subset$ $\Delta \mathfrak{s u}(2)$ and $\left[\mathfrak{m}_{1}, \mathfrak{m}_{2}\right]=0$. Thus we have the following non-zero symmetric triples to compute: [113] and [223]. We find that $[113]=15$, and we already computed $[223]=6$ above. We have $d_{1}=5, d_{2}=4, d_{3}=3$. As above, $b_{i}=12$. Hence, the Einstein equation is given by the system

$$
\frac{3 x_{3}}{2 x_{1}^{2}}-\frac{6}{x_{1}}=\frac{3 x_{3}}{4 x_{2}^{2}}-\frac{6}{x_{2}}=-\frac{5}{2 x_{3}}-\frac{x_{3}}{2 x_{2}^{2}}-\frac{5 x_{3}}{4 x_{1}^{2}} .
$$

Let $\alpha=\frac{x_{3}}{x_{1}}, \beta=\frac{x_{3}}{x_{2}}$, and simplify to get the following pair of equations: $0=$ $\beta^{2}-\frac{24}{5} \beta+2+\alpha^{2}$ and $0=\frac{11}{2} \alpha^{2}-12 \alpha+5+\beta^{2}$. This system has four solutions, with exactly two real positive solutions: $(\alpha, \beta) \cong(1.38166,1.03948)$ and $(\alpha, \beta) \cong(0.618099,0.562079)$, corresponding to two homogeneous Einstein metrics on $S U(3) \times S U(3) / \Delta_{2} S U(2)(\{\operatorname{id}\} \times U(1))$.

We next consider the homogeneous space $S U(3) \times S p(2) / \Delta_{1} S p(1)(\{\mathrm{id}\} \times S p(1))$, where $\operatorname{pr}_{1}\left(\Delta_{1} S p(1)\right)=S U(2)$ and $\operatorname{pr}_{2}\left(\Delta_{1} S p(1)\right)=S p(1) \times\{\operatorname{id}\}$. The isotropy representation can be seen to have four components: Let $\mathfrak{m}_{1}$ denote the orthogonal complement to $\mathfrak{u}(2)$ in $\mathfrak{s u}(3)$, and let $\mathfrak{m}_{2}$ be the complement to $\mathfrak{s p}(1) \oplus \mathfrak{s p}(1)$ in $\mathfrak{s p}(2)$. Then $\mathfrak{m}_{3}=\mathfrak{s u}(2) \oplus \mathfrak{s u}(2) \ominus \Delta \mathfrak{s u}(2)$, and $\mathfrak{m}_{4}=\mathfrak{u}(2) \ominus \mathfrak{s u}(2)$ (in $\mathfrak{s u}(3)$ ). We again have $\left[\mathfrak{m}_{3}, \mathfrak{m}_{4}\right]=0$, and $\left[\mathfrak{m}_{4}, \mathfrak{m}_{4}\right]=0$. We also know $\left[\mathfrak{m}_{1}, \mathfrak{m}_{2}\right]=0$ and $\left[\mathfrak{m}_{2}, \mathfrak{m}_{2}\right] \subset \mathfrak{s p}(1) \oplus \mathfrak{s} \mathfrak{p}(1)$. As above, $\left[\mathfrak{m}_{3}, \mathfrak{m}_{3}\right] \subset \Delta \mathfrak{s u}(2)$. Thus we have the following nonzero triples: [113], [114], and [223]. We already found [113] $=6$ above, and we see (after computing) that $[223]=6$ as well. If we consider the first example with $\lambda=1$, we find [114] $=12$. We have $d_{1}=d_{2}=4, d_{3}=3, d_{4}=1$, and $b_{i}=12$ for all $i$. Note this is exactly the data for the scalar curvature function we had in the example $S U(3) \times S U(3) / \Delta_{p, q} U(1) \Delta S U(2)$ for $\lambda=\lambda(p, q)=1$. Thus 
we can conclude that there are no $S U(3) \times S p(2)$-invariant Einstein metrics on $S U(3) \times S p(2) / \Delta_{1} S p(1)(\{\mathrm{id}\} \times S p(1))$.

The last space we need to consider is $S U(3) \times S p(2) / \Delta_{2} S p(1)(\{$ id $\} \times S p(1))$, where $\operatorname{pr}_{1}\left(\Delta_{2} S p(1)\right)=S O(3)$ and $\operatorname{pr}_{2}\left(\Delta_{2}(S p(1))\right)=S p(1) \times\{$ id $\}$. Here we again find ourselves combining information from the examples above. The isotropy representation has three summands: $\mathfrak{m}_{1}$ is the $Q$-orthogonal complement to $\mathfrak{s o}(3)$ in $\mathfrak{s u}(3), \mathfrak{m}_{2}$ is the complement to $\mathfrak{s p}(1) \oplus \mathfrak{s p}(1)$ in $\mathfrak{s p}(2)$, and $\mathfrak{m}_{3}$ is the complement to $\Delta \mathfrak{s p}(1)$ in $\mathfrak{s o}(3) \oplus \mathfrak{s p}(1)$. The non-zero symmetric triples are [113] $=15$ (found in the second example) and [223] $=6$ (found in the previous example). We have here $d_{1}=5, d_{2}=4, d_{3}=3$, and $b_{i}=12$ for all $i$. Note that this is exactly the data for the scalar curvature function we had in our second example, and so we obtain exactly two homogeneous Einstein metrics on $S U(3) \times S p(2) / \Delta_{2} S p(1)(\{$ id $\} \times S p(1))$.

Finally, we consider the case when $G / H$ is one of the spaces with abelian diagonal component in $H$. Existence of homogeneous Einstein metrics follows from the references provided in the Introduction, apart from four cases. In the last case we have to consider $T^{k}$-principal bundles, $k=1,2$, over $\mathbb{C} P^{1} \times \mathbb{C} P^{1} \times F_{3}$ presented as $S U(2) S U(2) S U(3) / T^{\prime}$, where $T^{\prime}<T^{4}$ is any 2-dimensional (resp. 3dimensional) compact subtorus of a maximal torus $T^{4}$ of $S U(2) S U(2) S U(3)$. We have $\Delta_{S U(2) S U(2) S U(3) / T^{\prime}}=\Delta_{S U(2) S U(2) S U(3) / T^{4}}$, hence by Theorem 2.4 .

$$
\Delta_{S U(2) S U(2) S U(3) / T^{\prime}}=\Delta_{S U(2) / U(1)} * \Delta_{S U(2) / U(1)} * \Delta_{S U(3) / T^{2}} * S^{1} .
$$

Since $\Delta_{S U(2) / U(1)}=\emptyset$ and $\Delta_{S U(3) / T^{2}}$ is disconnected, we conclude that

$$
\Delta_{S U(2) S U(2) S U(3) / T^{\prime}}
$$

is not contractible (cf. [Mi]). The existence of a homogeneous Einstein metric follows from Theorem 2.2. The other three cases can be treated in precisely the same manner.

This concludes the proof of the main theorem when $G$ is semisimple but not simple.

\section{REFERENCES}

[Ad] J. F. Adams: Lectures on Exceptional Lie Groups, Chicago Lectures in Mathematics, University of Chicago Press, Chicago, IL, (1996) MR1428422 (98b:22001)

[ADF] D. V. Alekseevsky, I. Dotti and C. Ferraris: Homogeneous Ricci positive 5-manifolds, Pacific J. Math. 175, 1-12, (1996) MR.1419469 (97k:53049)

[AlKi] D. V. Alekseevsky, B. N. Kimel'fel'd: Structure of homogeneous Riemann spaces with zero Ricci curvature, Func. Anal. Appl. 9, 97-102, (1975) MR0402650 (53:6466)

[Ar] A. Arvanitoyeorgos: New invariant Einstein metrics on generalized flag manifolds, Trans. Amer. Math. Soc. 337, 981-995, (1993) MR.1097162 (93h:53043)

[AlWa] S. Aloff, N. Wallach: An infinite family of distinct 7-manifolds admitting positively curved Riemannian structures, Bull. Amer. Math. Soc. 81, 93-97, (1975) MR0370624 (51:6851)

[BaHs] A. Back, W.-Y. Hsiang: Equivariant geometry and Kervaire spheres, Transactions Amer. Math. Soc. 304, 207-227 (1987) MR0906813 (88m:53073)

[Bes] A. L. Besse: Einstein Manifolds, Springer-Verlag, (1987) MR0867684 (88f:53087)

[Bo] S. Bochner: Curvature and Betti numbers, Ann. of Math. 49, 379-390, (1948) MR0025238(9:618d)

[Bö] C. Böhm: Homogeneous Einstein metrics and simplicial complexes, J. Diff. Geom. 67, 79-165, (2004) MR2153482

[BWZ] C. Böhm, M. Wang, W. Ziller: A variational approach for homogeneous Einstein metrics, GAFA 14, 681-733, (2004) MR2084976 (2005g:53074) 
[BoGa] C. P. Boyer, K. Galicki: On Sasakian-Einstein geometry, Internat. J. Math. 11, 873-909, (2000) MR 1792957 (2001k:53081)

[BGMR] C. P. Boyer, K. Galicki, B. M. Mann, E. G. Rees: Compact 3-Sasakian 7-manifolds with arbitrary second Betti number, Invent. Math. 131, 321-344, (1998) MR.1608567 (99b:53066)

[CDF] L. Castellani, R. D'Auria, P. Fré: $S U(3) \times S U(2) \times U(1)$ from $D=11$ supergravity, Nuclear Physics 239 B, 610-652, (1984) MR0749348 (86e:83052a)

[CRW] L. Castellani, L. J. Romans and N. P. Warner: A classification of compactifying solutions for $d=11$ supergravity, Nuclear Physics 241 B, 429-262, (1984) MR0757859 (86b:83039)

[DN] J. E. D'Atri, N. K. Nickerson: Divergence-preserving geodesic symmetry, J. Diff. Geom. 9, 251-262, (1979)

[DFN] R. D'Auria, P. Fré, P. van Nieuwenhuisen: $N=2$ matter coupled supergravity from compactification on a coset $G / H$ possessing an additional Killing vector, Phys. Lett. B 136, 347-353, (1984) MR0735731 (85d:83080)

[DK1] W. Dickinson, M. M. Kerr: The geometry of compact homogeneous spaces with two isotropy summands, preprint (2002)

[DK2] W. Dickinson, M. M. Kerr: Einstein metrics on compact homogeneous spaces, preprint (2002)

[Dy1] E. B. Dynkin: Maximal subalgebras of the classical groups, Transl. Am. Math. Soc. Series 2, Vol. 6, 245-378, (1957) MR0049903 (14:244d)

[Dy2] E. B. Dynkin: Semisimple subalgebras of semisimple Lie algebras, Transl. Am. Math. Soc. Series 2, Vol. 6, 111-244, (1957) MR0047629 (13:904c)

[He] J. Heber: Noncompact homogeneous Einstein spaces, Invent. Math. 133, 279-352, (1998) MR.1632782 (99d:53046)

[Hi] D. Hilbert: Die Grundlagen der Physik, Nachr. Akad. Wiss. Gött., 395-407, (1915)

[Je1] G. Jensen: Homogeneous Einstein spaces of dimension 4, J. Diff. Geom. 3, 309-349, (1969) MR0261487 (41:6100)

[Je2] G. Jensen: The scalar curvature of left invariant Riemannian metrics, Indiana U. Math. J. 20, 1125-1144, (1971) MR0289726(44:6914)

[Je3] G. Jensen: Einstein metrics on principal fibre bundles, J. Diff. Geom. 8, 599-614 (1973) MR0353209(50:5694)

[Jo] D. Joyce: Compact manifolds with special holonomy, Oxford Mathematical Monographs, Oxford University Press (2000) MR.1787733 (2001k:53093)

[Ke] M. M. Kerr: Some new homogeneous Einstein metrics on symmetric spaces, Trans. Amer. Math. Soc. 348, 153-172, (1996) MR1327258 (96f:53064)

[Ki] M. Kimura: Homogeneous Einstein Metrics On Certain Kähler $C$-Spaces, Adv. Stud. Pure Math., 18-I, Recent topics in differential and analytic geometry, 303-320, (1990) MR.1145261 (93b:53039)

[Kl] S. Klaus: Einfach-zusammenhängende Kompakte Homogene Räume bis zur Dimension Neun, Diplomarbeit, Johannes Gutenberg Universität (1988)

[Ko] S. Kobayashi: Topology of positively pinched Kähler manifolds, Tohoku Math. J. 15, 121-139, (1963) MR0154235 (27:4185)

[Kosz] J. L. Koszul: Sur la forme hermitienne canonique des espaces homogènes complexes, Can. J. of Math. 7, 562-576, (1955) MR0077879(17:1109a)

[KS1] M. Kreck, S. Stolz: A diffeomorphism classification of 7-dimensional homogeneous Einstein manifolds with $\mathrm{SU}(3) \times \mathrm{SU}(2) \times \mathrm{U}(1)$-symmetry, Ann. of Math. 127, 373-388, (1988) MR0932303 (89c:57042)

[KS2] M. Kreck, S. Stolz: Some nondiffeomorphic homeomorphic homogeneous 7-manifolds with positive sectional curvature, J. Diff. Geom. 33, 465-486, (1991); correction, J. Diff. Geom. 49, 203-204, (1998) MR1094466 (92d:53043)

[Kr] B. Kruggel: Quotienten halbeinfacher, kompakter Liescher Gruppen; Eine Klassifikation bis zur Dimension Zwölf, Diplomarbeit, Heinrich-Heine-Universität Düsseldorf (1993)

[LeWa] C. LeBrun, M. Y. Wang: Surveys in differential geometry VI: essays on Einstein manifolds, eds. C. LeBrun, M. Y. Wang, International Press, (1999) MR:1798603 (2001f:53003)

[Mi] J. Milnor: Construction of Universal Bundles, II, Ann. of Math. 63, 430-436, (1956) MR0077932(17:1120a) 
[NiRo] Yu. G. Nikonorov and E. D. Rodionov: Compact 6-dimensional homogeneous Einstein manifolds, Dokl. Math. RAS 336, 599-601, (1999) MR1721419 (2000g:53054)

[Ni1] Yu. G. Nikonorov: On one class of homogeneous compact Einstein manifolds, Siberian Math. J. 41, No. 1, 168-172, (2000) MR.1756487 (2001i:53075)

[Ni2] Yu. G. Nikonorov: Compact homogeneous Einstein 7-manifolds, Geom. Dedicata 109, 7-30, (2004) MR 2113184

[On] A. L. Onishchik: Topology of Transitive Transformation Groups, Barth, (1994)

[PP] D. Page, C. Pope: New squashed solutions of $d=11$ supergravity, Phys. Lett. 147B, 55-60, (1984) MR0768319 (86h:83062)

[Sa] Y. Sakane: Homogeneous Einstein Metrics on flag manifolds, Lobach. Jour. of Math. 4, 71-87, (1999) MR:1743146 (2000m:53069)

[Tia] G. Tian: Kähler-Einstein metrics with positive scalar curvature, Invent. Math. 130, 1-37, (1997) MR1471884 (99e:53065)

[Wa1] M. Wang: Some examples of homogeneous Einstein manifolds in dimension seven, Duke Math. J. 49, 23-28, (1982) MR0650366 (83k:53069)

[Wa2] M. Wang: Einstein metrics and quaternionic Kähler manifolds, Math. Z. 210, no. 2, 305-325 (1992) MR 1166528 (93h:53046)

[WZ1] M. Wang, W. Ziller: On normal homogeneous Einstein manifolds, Ann. Scient. Éc. Norm. Sup., $4^{e}$ série t. 18, 563-633, (1985) MR0839687 (87k:53113)

[WZ2] M. Wang, W. Ziller: Existence and non-existence of homogeneous Einstein metrics, Invent. Math. 84, 177-194, (1986) MR0830044 (87e:53081)

[WZ3] M. Wang, W. Ziller: Einstein metrics on principal torus bundles, J. Diff. Geom. 31, 215-248, (1990) MR1030671 (91f:53041)

[Wo] J. A. Wolf: The geometry and structure of isotropy irreducible homogeneous spaces, Acta Math. 120, 59-148 (1968); correction, Acta Math. 152, 141-142 (1984). MR0223501 (36:6549)

[Yau] S. T. Yau: On the Ricci curvature of a compact Kähler manifold and the complex Monge-Ampère equation I, Comm. Pure Appl. Math. 31, 339-411, (1978) MR0480350 (81d:53045)

[Zi] W. Ziller: Homogeneous Einstein metrics on spheres and projective spaces, Math. Ann. 259, 351-358, (1982) MR0661203 (84h:53062)

Mathematisches Institut, Universität Münster, Einsteinstr. 62, 48149 MÜnster, GerMANY

E-mail address: cboehm@math.uni-muenster.de

Department of Mathematics, Wellesley College, 106 Central St., Wellesley, MasSACHUSETTS 02481

E-mail address: mkerr@wellesley.edu 